\title{
Nanoring as a magnetic or electric field sensitive nano-antenna for near-field optics applications
}

\author{
M.A. Suarez *, T. Grosjean, D. Charraut, D. Courjon \\ Institut FEMTO-ST, Département d'Optique P.M. Duffieux, UMR 6174, 16 route de Gray, 25030 Besançon cedex, France
}

\begin{abstract}
In this paper we investigate, in the visible range, the behavior of the electromagnetic near-field, perturbed by a metal nanoring. The vectorial study which is based on FDTD simulation, consists of comparing in the near zone, the electric and magnetic field distributions while interacting with the metal nanoring, according to the incident polarization state. Both image distributions and chromatic spectra are computed and analyzed. The purpose of this work is the enhancement of either the electric or the magnetic emission/detection capability of nanoring according to illumination conditions. Such nano-objects can be seen as particular selective nanodevices playing the role of nano-antennas usable in near-field microscopy as an alternative solution to usual tips.
\end{abstract}

Keywords: FDTD simulation; Nano-antenna; Near-field; Radial polarization; Azimuthal polarization

\section{Introduction}

One of the critical components in near-field optics is the nano-collector/nano-emitter. After twenty years of existence of optical near-field microscopy, we cannot consider that the problem has been efficiently solved. Despite a few emerging experimental solutions [1-5] and a great deal of theoretical works [6-10], the problem of understanding the optical mechanism both at the apex and between apex and sample is still questionable. Another aspect of the detection (emission) process is the type of signal which is detected. If in far-field the problem is reduced to the detection of the Poynting vector flux, in near-field optics, the notion of Poynting vector is not relevant since there is no energy flux transmitted in the non-radiative regime (evanescent waves). Therefore, the usual far-field assertion saying that the detection of the Poynting vector flux describes completely the behavior of the electromagnetic flux is

\footnotetext{
* Corresponding author. Tel.: +33 381666624; fax: +333816666423. E-mail address: miansu@mixmail.com (M.A. Suarez).
}

meaningless in the near zone, because the notion of energy flux is associated with a particular direction of propagation through a given surface. In the near zone, the fields are both evanescent and propagative and although a Poynting vector can be defined, its flux cannot be determined since no privileged direction can be deduced. This physical fact raises an open question: what does the nano-collector detect in the near zone? In the far-field, in free space, the Poynting vector can be written either in terms of electric or magnetic field, that is, except for some phase terms, there is equivalence between electric and magnetic fields. In the near zone, this equivalence is broken and the electric field mapping generally does not coincide with the magnetic one.

In this study, we will discuss the particular case of light interaction with a metal nanoring, the dimension of which is of the order of magnitude of the wavelength. Precedent works by Aizpurua et al. [11], Prodan et al. [12], and Dereux et al. [13-15] have reported the behavior of such annular structure. We will investigate several physical situations namely (i) the illumination of the nanoring with 
linear, radial and azimuthal polarizations (ii) the study of field behavior according to the wavelength.

The work presented here is theoretical and numerical. It is based on the use of the well-known Finite-Difference Time-Domain (FDTD) [16].

The final aim is to a priori define nano-antennas exhibiting strong differences between electric and magnetic emission/detection capabilities, in order, to use them in a second step in near-field microscopy as a new generation of nano-emitters/nanocollectors.

\section{Modeling protocol}

A great deal of numerical methods have been carried out for the last decades to determine the electric and the magnetic field behavior in the case of visible light interacting with micro- and submicro-structures. One of the most promising methods is the so-called FDTD which can faithfully mimic real (3D) situations. However, despite of its performances, the FDTD method is characterized by a few inherent drawbacks connected to the dispersion law, the cubic mesh and some spurious effects of reflection on the computing space boundaries. The FDTD program we have used is a commercial one.

\subsection{Dispersion law}

The retained metal is gold because of its absorption properties in the visible range (plasmon generation). In order to reproduce as faithfully as possible the actual experimental case we have first of all determined the dispersion curve $\varepsilon(\omega)$ of the metal chosen for future experiments. Instead of Drude or Lorentz dispersion laws which are usually used, but strongly diverge below $650 \mathrm{~nm}$ (see Fig. 1(b)), we have used the most generic dispersion law derived from Sellmeier coefficients [17]:

$\varepsilon(\omega)=\varepsilon_{\infty}+\sum_{k=1}^{4} \frac{\delta_{k}}{-a_{k} \omega^{2}-j b_{k} \omega+c_{k}}$ where $\delta_{k}, a_{k}, b_{k}$ and $c_{k}$ are coefficients allowing the fine fitting of the dispersion law. This relation is merely a linear superposition of resonance terms. According to the table of physical constants [18], the coefficients of Eq. (1) can be easily determined leading to a good agreement between experimental data and theoretical curve far beyond $650 \mathrm{~nm}$. The fitting procedure is borrowed from Newton method.

\subsection{Computing conditions}

The FDTD solves Maxwell's equations by approximating both time and space derivatives with finite-difference forms. The technique consists of dividing the space to be analyzed into a mesh of small cubic cells characterized both by their local electromagnetic properties and by the couple of vectors $\mathbf{E}$ and $\mathbf{H}$. The electric and magnetic field components are then interlaced in all three spatial dimensions. The upper bound of the cell size is not larger than one tenth of a wavelength in order to ensure a sufficient sampling of the fields according to the rules of validity of FDTD (geometry and local material properties). The fields are expressed in the gaussian system in which $\mathbf{E}$ and $\mathbf{H}$ have the same unity.

A specific problem of the FDTD method is that the mesh is necessary composed of elementary cubic cells, the walls of which correspond to the finite-difference along the $x, y, z$ directions. In the case of objects exhibiting curved lines like disks and rings, the number of elementary cells must be as large as possible in order to limit numerical artifacts.

Another critical point is the boundary conditions at the spatial limits of the computational domain. One of the most efficient boundary conditions that cancels any outward propagating energy is the so-called Perfectly Matched Layer (PML) [19]. In such a method both electric and magnetic conductivities are introduced in such a way that the wave impedance remains constant, absorbing the energy without inducing reflections. However, because most PML implementations do not process the evanescent com-
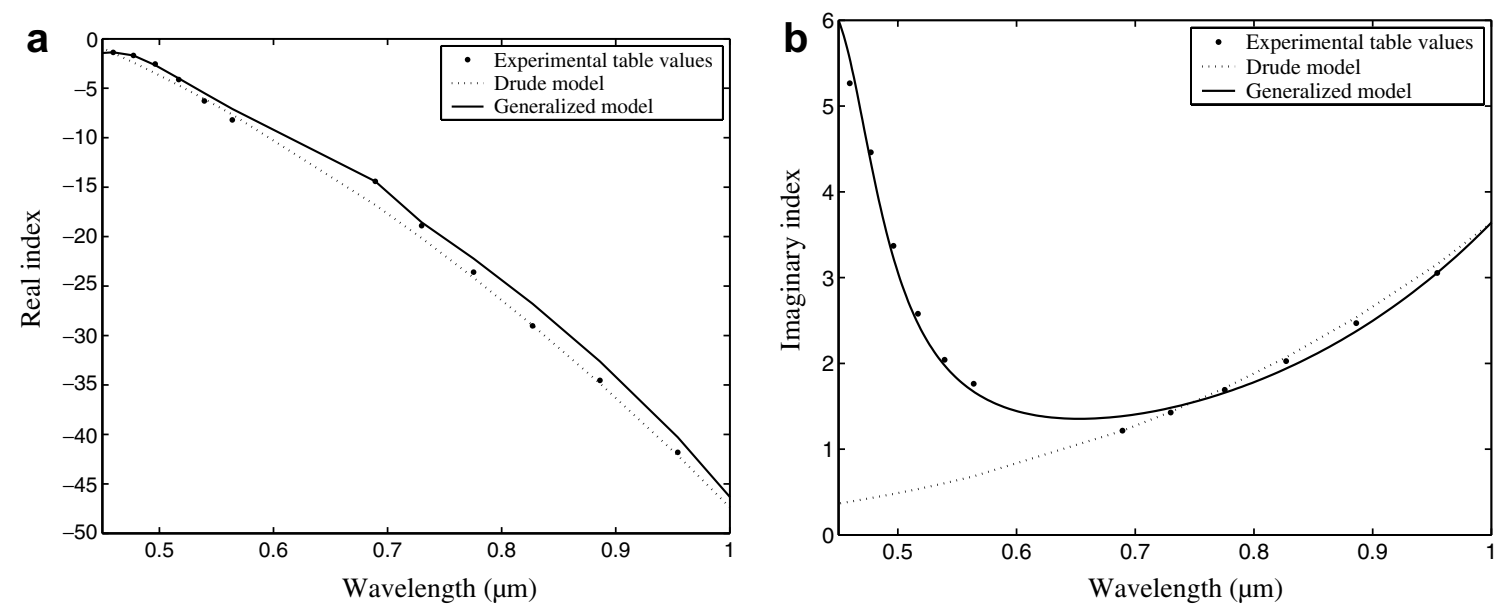

Fig. 1. Curves of dispersion of $\varepsilon$ in the visible range. (a) Real part. (b) Imaginary part. 


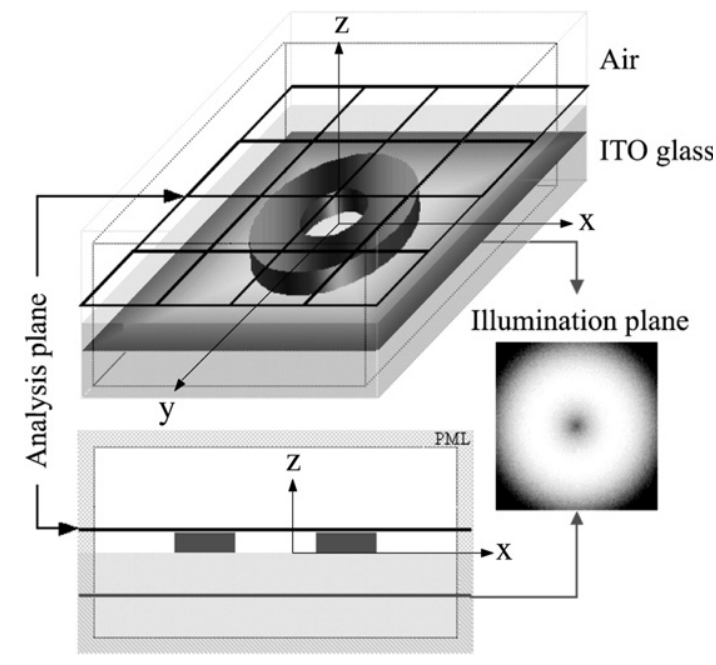

Fig. 2. Nanoring and computational domain.

ponents of the field, it is necessary to prevent parasitic effects due to evanescent wave reflection by adding a free gap between the structure and the domain boundaries.

\subsection{Numerical parameters and initial conditions}

The parameter choice is deduced both from experimental constraints about acceptable object size and from a set of critical convergence tests. The structure is a $3 \mathrm{D}$ gold submicrometer ring of $500 \mathrm{~nm}$ outer radius, $200 \mathrm{~nm}$ inner radius and $100 \mathrm{~nm}$ thickness deposited on ITO glass (see Fig. 2). According to FDTD accuracy constraints the computational volume is chosen as $1840 \mathrm{~nm} \times 1840 \mathrm{~nm} \times 945$ $\mathrm{nm}$ including a $420 \mathrm{~nm}$ free space (about the working half wavelength). The selected mesh takes into account the available computer memory leading to a computing cubic cell of $\Delta x=\Delta y=5 \mathrm{~nm}, \Delta z=7 \mathrm{~nm}$. The resulting computational space is composed of $416 \times 416 \times 183$ points. According to the validity condition of FDTD the time step $\Delta t$ equals $5.5 \times 10^{-3}$ fs.

\section{Illumination conditions}

In a general way, the excitation (electric) field can be written as follows:

$\mathbf{E}_{0}\left(x, y, z_{\mathrm{p}}, t\right)=\mathbf{e}\left(x, y, z_{\mathrm{p}}\right) \cdot f(t)$

where $\left(x, y, z_{\mathrm{p}}\right)$ are the spatial cartesian coordinates defining the illumination plane (see Fig. 2) and $t$ is the time coordinate. Vector e defines the spatial behavior of $\mathbf{E}_{0}$ (complex field amplitude) whereas function $f$ defines its temporal behavior. It turns out that these two parameters play a key role in the interaction process. Let us note that the phase of vector $\mathbf{e}$ has been set constant in the illumination plane.

\subsection{Spatial behavior and polarization of the fields}

The spatial behavior of $\mathbf{E}_{0}$ depends strongly on its polarization state. Taking into account the particular geometry of the structure, three specific polarizations have been retained for the incident light: the linear polarization and the highly symmetrical radial and azimuthal ones.

\subsubsection{Linear polarization}

The light beam is polarized in the $x$ direction. In order to limit the parasitic effects on the computation boundaries, we have opted for a gaussian beam profile as shown in Fig. 3.

\subsubsection{Radial polarization}

In this case, the electric field radially vibrates in every space point from the optical axis. This particular case is interesting because it exhibits axial symmetry and leads generally to electric and magnetic fields which can be simply analytically described $[20,21]$. Fig. 4(a)-(c) shows the electric field amplitude $\left(\left|\mathbf{e}\left(x, y, z_{\mathrm{p}}\right)\right|\right)$ and the components $e_{x}$ and $e_{y}$, respectively.

\subsubsection{Azimuthal polarization}

In that situation the magnetic field radially vibrates. Similarly to the case of radial polarization, azimuthal polarization exhibits axial symmetry. Fig. 4(d)-(f) shows the electric field amplitude $\left(\left|\mathbf{e}\left(x, y, z_{\mathrm{p}}\right)\right|\right)$ and the components $e_{x}$ and $e_{y}$, respectively.

\subsection{Time and spectral behavior}

One of the aims of this study is to determine the spectral behavior of the field interacting with the nanoring antenna. Since FDTD method solves the space and time Maxwell equations, we can easily choose the time dependence $f(t)$ of the excitation field (see Eq. (2)). For instance, if $f(t)$ is constant, the excitation regime is permanent and monochromatic. Conversely, if it is time limited, the spectrum will cover a more or less wide wavelength range. Here, $f(t)$ is defined as a sinusoidal function apodized by means of a gaussian envelop. We have:

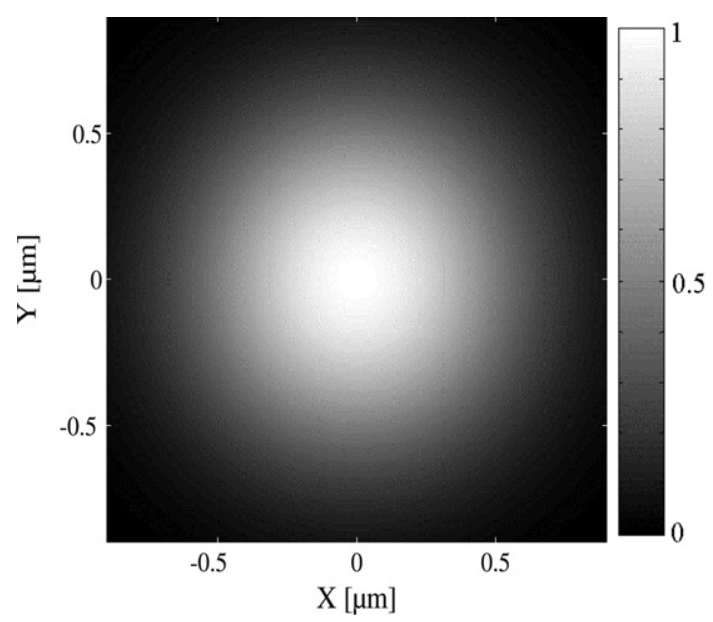

Fig. 3. Electric field amplitude for linear polarization. 

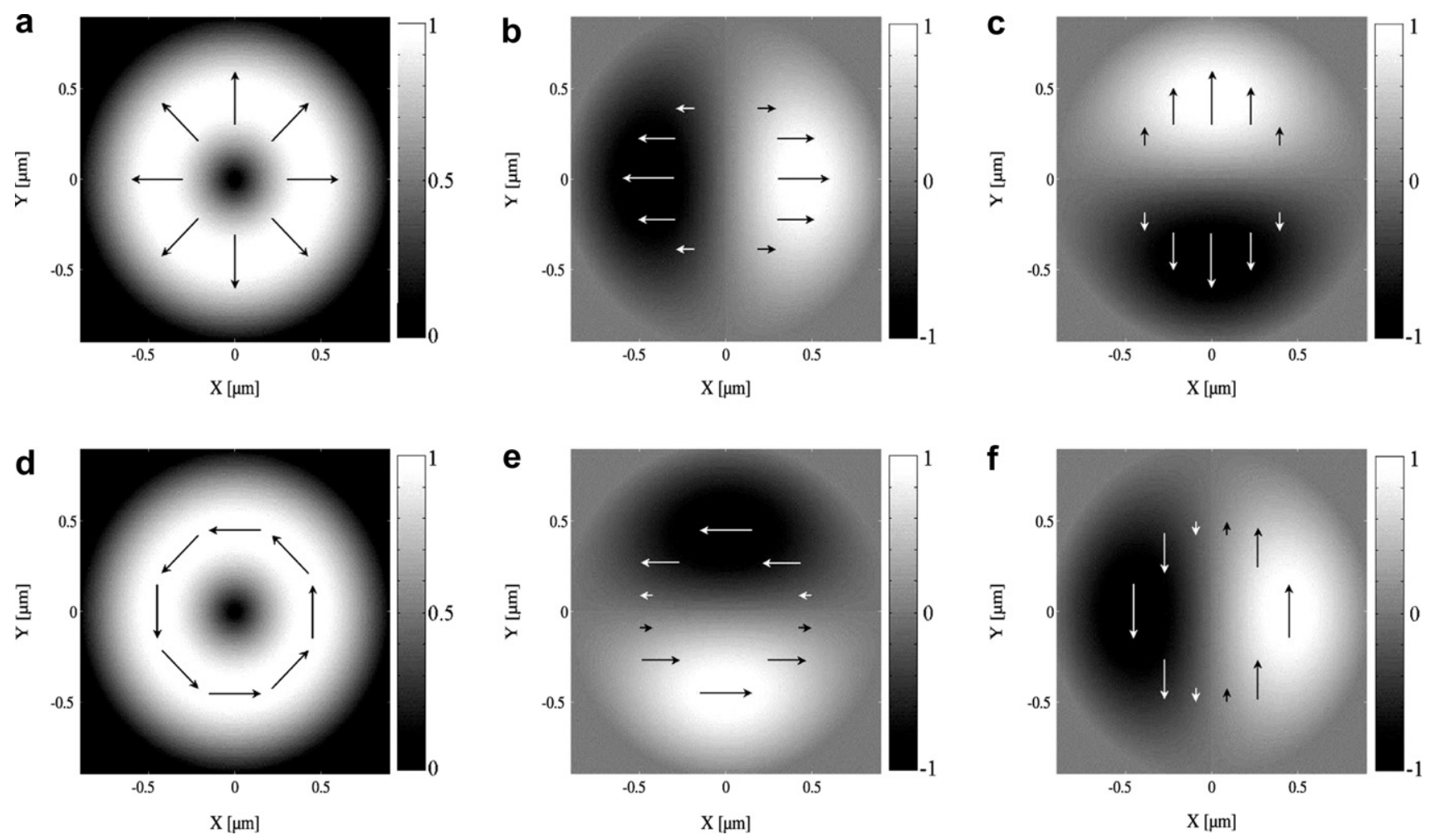

Fig. 4. Electric field amplitude for (a) radial polarization and (d) azimuthal polarization; (b,c) and (e,f) are the $x$ and $y$ field components respectively. The arrows shows the direction of the electric field oscillation.

$f(t)=\exp \left[-\left(\frac{t-t_{\mathrm{d}}}{\tau}\right)^{2}\right] \sin \left[\omega_{\mathrm{c}} t\right]$

where $\omega_{\mathrm{c}}$ is the central excitation angular frequency, $\tau$ defines the pulse time duration and $t_{\mathrm{d}}$ is a time delay. In this study, we have chosen $\tau=1.9762 \mathrm{fs}$ and $\omega_{\mathrm{c}}=510 \mathrm{THz}$. Such an excitation pulse leads to a gaussian spectrum ranging from 350 to $670 \mathrm{THz}(450-850 \mathrm{~nm})$. The computational time is chosen as long as necessary in such a way that after passing through the structure the field completely vanishes in the computational domain $\left(\approx 90 \mathrm{fs}, 2^{14} \Delta t\right)$.

\section{Results and discussion}

In order to point out the specific effect of the nanoring, the fields are computed with and without ring. In what follows, the electric and magnetic fields $\mathbf{E}$ and $\mathbf{H}$ will be called $\mathbf{g}_{V}(x, y, z, t)$ without the nanoring and $\mathbf{g}_{R}(x, y, z, t)$ with the nanoring. The fields $\mathbf{g}_{V}(x, y, z, t)$ and $\mathbf{g}_{R}(x, y, z, t)$ are computed in the same analysis plane $\left(x, y, z_{0}\right)$ (see Fig. 2). To be as close as possible to experimental conditions in near field, this plane is located very near to the nano-antenna ( $7 \mathrm{~nm}$ from the top of the ring, corresponding to one computation spatial step).

The spectral distributions of the fields in the analysis plane are numerically obtained by means of the following expression (numerical Fourier transform):

$G_{i}\left(x, y, z_{0}, \omega\right)=\left|\sum_{n} \mathbf{g}_{i}\left(x, y, z_{0}, n \cdot \Delta t\right) \exp (-\mathrm{j} \omega n \cdot \Delta t)\right|^{2}$ where $i$ stands for the indices $R$ or $V$. Let us note that $G_{i}\left(x_{0}, y_{0}, z_{0}, \omega\right)$ is the chromatic spectrum at the point $\left(x_{0}, y_{0}, z_{0}\right)$ and $G_{i}\left(x, y, z_{0}, \omega_{0}\right)$ is the intensity distribution in the analysis plane at frequency $\omega_{0}$.

\subsection{Chromatic spectrum}

For this purpose, the spectrum of the diffracted fields has been studied at five points $(A-E)$ in the analysis plane (Fig. 5(a)). This choice can be justified from preliminary computations and from the fact that first, plasmon exaltations are generally generated at discontinuities such as walls, second, the center plays a privileged role, since the nano-antenna will be used as imaging sensor. The normalized spectrum $S$ at each point is defined as:

$S\left(x_{i}, y_{i}, z_{0}, \omega\right)=N\left(x_{i}, y_{i}, z_{0}\right) \frac{G_{R}\left(x_{i}, y_{i}, z_{0}, \omega\right)}{G_{V}\left(x_{i}, y_{i}, z_{0}, \omega\right)} \quad i=A$ to $E$

where $N\left(x_{i}, y_{i}, z_{0}\right)=\max \left[G_{V}\left(x_{i}, y_{i}, z_{0}, \omega\right)\right]$ is a normalization function of $G_{V}$. Function $N$ allows us to compare quantitatively the spectra calculated in points $A-E$. This procedure takes into account the spatial behavior of both the spectral and amplitude distributions of the incident beam in the analysis plane.

Fig. 5 shows function $S\left(x_{i}, y_{i}, z_{0}, \omega\right)$ for $(\mathrm{b}, \mathrm{c})$ linear, $(\mathrm{d}, \mathrm{e})$ radial and (f,g) azimuthal polarizations. With the highly symmetric radial and azimuthal polarizations, only the spectra at points A, B and C are displayed (Fig. 5(d)-(g)). 
a
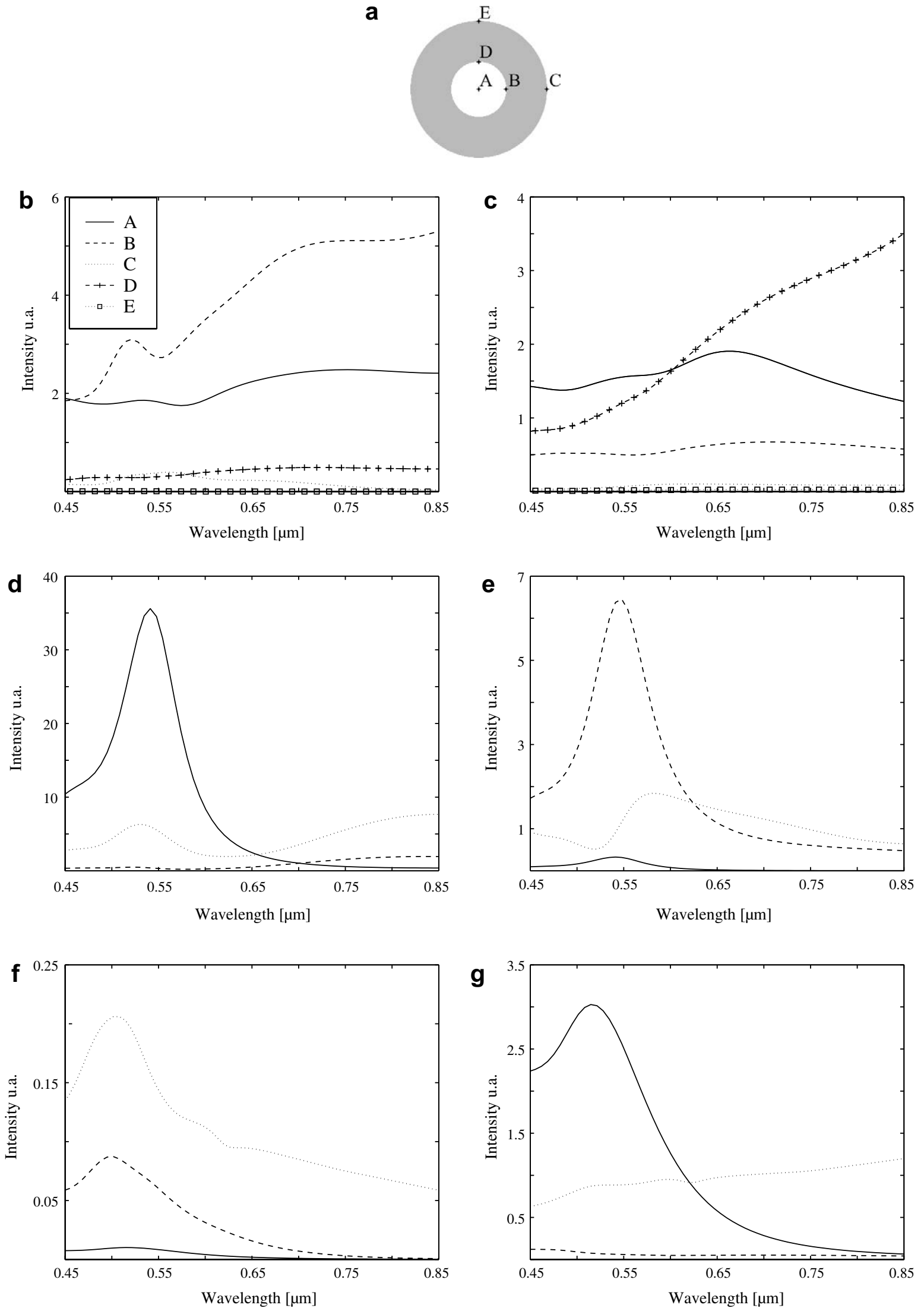

Fig. 5. (a): Position of the analysis points $A-E$ with respect to nanoring. (b,d,f) spectral curves $S$ for the electric field. (c,e,g) Spectral curves $S$ for the magnetic field. (b,c) Linear polarization directed along (AC); (d,e) radial polarization; (f, g) azimuthal polarization. 


\subsection{Field intensity distribution}

Let us consider the following equation:

$F\left(x, y, z_{0}, \omega_{0}\right)=G_{R}\left(x, y, z_{0}, \omega_{0}\right)-G_{V}\left(x, y, z_{0}, \omega_{0}\right)$

where $\omega_{0}$ is a specific frequency in the spectra of Fig. 5. This subtraction improves the understanding of the field behavior which is no longer spoiled by the nonuniform incident field. It can be easily experimentally implemented by first, recording the field intensity $G_{V}$ (without the nanoring) and second, subtracting for each point the signal $G_{R}$ (with the nanoring). Let us note that another way leading to an approximative result could be easily experimentally reproduced by modulating the tip-to-sample distance (near-field and far-field detection).
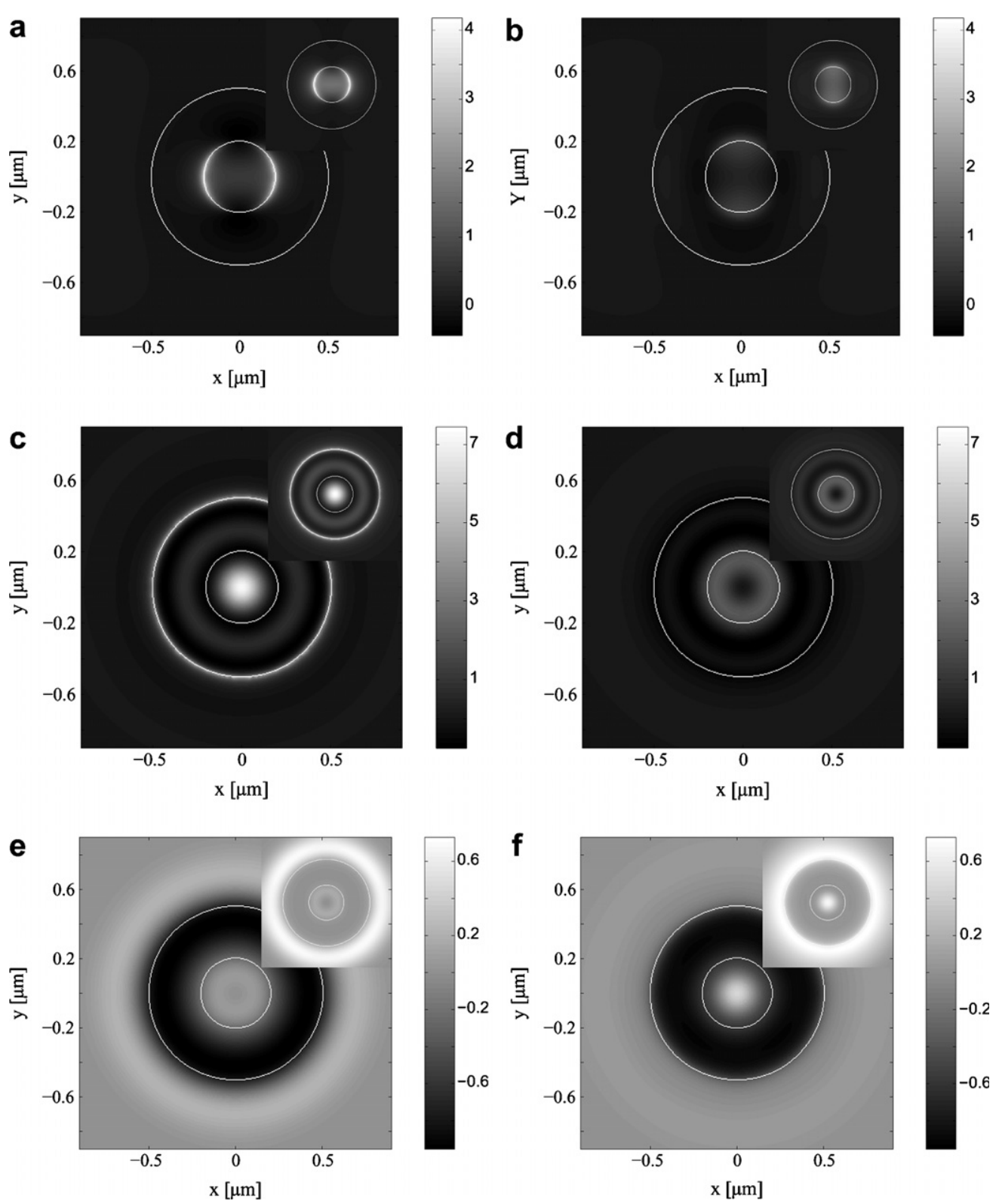

Fig. 6 exhibits for each case a main image $(F)$ and an inset $\left(G_{R}\right)$; this one is the raw detected signal which takes into account the incident field distribution.

\subsection{Discussion}

In linear polarization (Fig. 5(b) and (c)), a strong exaltation is observed at point B (for electric field) and at point D (for magnetic field) for the highest wavelengths. Such a variation points out the presence of a plasmon mode of the structure for the considered wavelength range. The absence of a resonance peak can be explained by the fact that the plasmon mode is subject to a strong dispersion. Fig. 6(a) and (b) depict the variation of $F$ at $\lambda_{0}=752 \mathrm{~nm}$. The electric and magnetic fields are mostly concentrated on the

Fig. 6. Depiction of function $F\left(x, y, z_{0}, \omega_{0}\right)$ for $(\mathrm{a}, \mathrm{c}, \mathrm{e})$ electric field and $(\mathrm{b}, \mathrm{d}, \mathrm{f})$ magnetic field, for three polarization states: $(\mathrm{a}, \mathrm{b})$ linear $\left(\lambda_{0}=752 \mathrm{~nm}\right)$, $(\mathrm{c}, \mathrm{d})$ radial $\left(\lambda_{0}=541 \mathrm{~nm}\right)$ and $(\mathrm{e}, \mathrm{f})$ azimuthal $\left(\lambda_{0}=514 \mathrm{~nm}\right)$ polarizations. The figure insets show the intensity distribution $G_{R}$ in the analysis plane. 
inner wall of the structure and no field confinement is observed at the center of the ring. Such a field behavior has been previously observed on a metal torus [15] as well as on metal-coated aperture probes [22] and metal nanocylinders [14].

In Refs. [15,14], it has been shown that effective electric or magnetic dipole moments can be associated to such field distributions, depending on wavelength and structure dimensions. Because nanocylinders are bidimensional nanorings, we expect the same properties in this configuration. Therefore, the nanoring can be a transverse magnetic field nano-collector, as experimentally shown for nanocylinderlike structures [13].

In the case of radial polarization (Fig. 5(d) and (e)), a strong resonance is observed at $\lambda_{0}=541 \mathrm{~nm}$ for both electric and magnetic fields. Fig. 6(c) and (d) show that this resonance is characterized by a strong electric field concentration in the center zone and a magnetic field which spreads out inside the ring. A fine analysis of the electric field components shows that the exalted electric field confinement is longitudinally polarized (along $z$ ). The enhancement factor of electric field is 2.98 times larger than the one of the magnetic field. In azimuthal polarization (Fig. 5(f) and (g) and Fig. 6(e) and (f)), the inverse situation is observed. It means that the magnetic field is confined and exalted in the center whereas the electric field keeps out the confinement zone at resonance frequency $\left(\lambda_{0}=\right.$ $514 \mathrm{~nm}$ ). In this configuration, the magnetic field hot spot is longitudinally polarized and the magnetic field increase is 3.5 times larger that the electric field one. These two orthogonal polarizations lead to symmetrical situations for the electric and magnetic fields emitted by the nanoantenna.

In the radial case, the narrow spectral resonance is associated to a low dispersive plasmon mode of the nanoantenna, which is observed with infinitely long cylinders [14]. In a first approximation, it can be associated to a dipole moment longitudinally oriented. We note that such a behavior is encountered with the radially polarized confined field without ring $[20,23]$. The effect of the nanoring is to strongly increase the longitudinal dipole moment as shown in Fig. 5(d) which points out a longitudinal-field enhancement by a factor 35.5 with the nano-antenna. This property is confirmed in Table 1.

Therefore this configuration leads to a longitudinallypolarized electric field nanosource. Reciprocally, the resonant nanoring associated to a radial polarizer becomes a longitudinal electric field nano-collector. Since no azimuth-

Table 1

Ratio between the respective maxima of the transverse and longitudinal fields with and without nanoring

\begin{tabular}{lll}
\hline & Without ring $G_{V}$ & With the ring $G_{R}$ \\
\hline $\begin{array}{l}\text { Radial polarization } \\
\max \left[E_{z}^{2}\right] / \max \left[E_{t}^{2}\right]\end{array}$ & 0.3 & 3.05 \\
$\begin{array}{l}\text { Azimuthal polarization } \\
\max \left[H_{z}^{2}\right] / \max \left[H_{t}^{2}\right]\end{array}$ & 0.26 & 1.12 \\
\hline
\end{tabular}

ally-polarized plasmon mode is carried by bidimensional nanorings, the plasmonic origin of the field exaltation in Fig. 5(f) and (g) is disputable. This is confirmed by the fact that the intensity of the field generated by the nanoantenna is as high as the incident field intensity (Fig. 6(e) and (f)). Nevertheless, a non-negligible longitudinally-oriented magnetic moment is generated at the center of the ring. This configuration originates either a longitudinally polarized magnetic nanosource or a collector mostly sensitive to the longitudinal magnetic field. In the latter case, the nano-antenna should be associated to an azimuthal polarizer.

Let us note that first, the polarization appears to be the central parameter for emission and detection properties of nano-antennas. This point has been already observed for a specific bow-tie and dipolar antenna [24,25]. Second, it is possible to imagine new kind of nanosources able to emit either a confined magnetic field or a confined electric one. The operation consists merely in toggling from radial to azimuthal polarization and choosing the suitable spectral band. Another solution could consist in choosing a geometry in such a way that both electric and magnetic field are exalted for two different wavelengths. The nanosource will be either an electric emittor or a magnetic one according to the wavelength.

\section{Conclusion}

In this article, we have described and studied a simple loop nano-antenna usable in various domains such as near-field microscopy, near-field nanolithography, biology, etc. These new sources can behave either as magnetic or electric field emitters according to the incident polarization (radial or azimuthal). This behavior is only visible and usable in the near-field zone where there is no identity between magnetic and electric field distributions. Let us note that similar conclusions can be drawn in the case of nanocollection mode. We hope that this work will open a new field of research in magnetic-oriented or electric-oriented nano-characterization. Micro and nanofabrication techniques allowing reproducible nanoring realizations are currently developed for further experimental studies.

\section{References}

[1] F. Zenhausern, M.P. O'Boyle, H.K. Wickramasinghe, Appl. Phys. Lett. 65 (1994) 1623.

[2] P. Gleyzes, A.C. Boccara, R. Bachelot, Ultramicroscopy 57 (1995) 318.

[3] C. Ecoffet, R. Bachelot, D. Deloeil, P. Royer, D.J. Lougnot, Synthetic Met. 124 (2001) 29.

[4] A. Naber, D. Molenda, U.C. Fischer, H.J. Maas, C. Höppener, N. Lu, H. Fuchs, Phys. Rev. Lett. 89 (2002) 210801-1.

[5] O. Sqalli, I. Utke, P. Hoffmann, F. Marquis-Weible, J. Appl. Phys. 92 (2002) 1078.

[6] D. Van Labeke, F.I. Baida, D. Barchiesi, D. Courjon, Opt. Commun. 114 (1995) 470.

[7] R. Carminati, J.J. Greffet, Opt. Commun. 116 (1995) 316.

[8] O.J.F. Martin, J. Microsc. 194 (1999) 235. 
[9] A. Bouhelier, J. Renger, M. Beversluis, L. Novotny, J. Microsc. 210 (2003) 220.

[10] T. Laroche, F.I. Baida, D. Van Labeke, JOSA B. 22 (2005) 1045.

[11] J. Aizpurua, P. Hanarp, D.S. Sutherland, M. Käll, G.W. Bryant, F.J. Garcia de Abajo, Phys. Rev. Lett. 90 (2003) 057401-1.

[12] E. Prodan, C. Radloff, N.J. Halas, P. Nordlander, Science 302 (2003) 419.

[13] E. Devaux, A. Dereux, E. Bourillot, J.C. Weeber, Y. Lacroute, J.P. Goudonnet, C. Girard, Phys. Rev. B. 62 (2000) 10504.

[14] U. Schröter, A. Dereux, Phys. Rev. B 64 (2001) 125420/1.

[15] A. Mary, A. Dereux, T. Ferrell, Phys. Rev. B 72 (2005) 155426/1.

[16] A. Taflove, S. Hagness, Computational Electrodynamics: The FiniteDifference Time-Domain Method, Third ed., Artech House, Boston, MA, 2005.
[17] K.V. Namjoshi, S.S. Mitra, Phys. Rev. B. 9 (1974) 815.

[18] E. Palik, Handbook of Optical Constants of Solids, Academic Press, 1985.

[19] J.P. Berenger, J. Comput. Phys. 114 (1994) 185.

[20] K.S. Youngworth, T.G. Brown, Opt. Express 7 (2000) 77.

[21] T. Grosjean, A. Sabac, D. Courjon, Opt. Commun. 252 (2005) 12.

[22] L. Novotny, D.W. Pohl, B. Hecht, Ultramicroscopy 61 (1995) 1.

[23] L. Novotny, M.R. Beversluis, K.S. Youngworth, T.G. Brown, Phys. Rev. Lett. 86 (2001) 5251.

[24] P.J. Schuck, D.P. Fromm, A. Sundaramurthy, G.S. Kino, W.E. Moerner, Phys. Rev. Lett. 94 (2005) 017402/1.

[25] P. Mühlschlegel, H.J. Eisler, O.J.F. Martin, B. Hecht, D.W. Pohl, Science 308 (2005) 1607. 